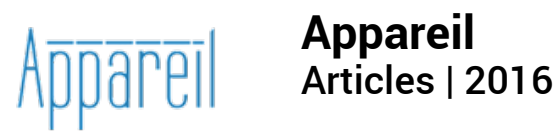

\title{
Le travail de Rithy Panh : un appareil funéraire
}

\section{Martine Lefeuvre-Déotte}

\section{(2) OpenEdition}

\section{Journals}

\section{Electronic version}

URL: http://journals.openedition.org/appareil/2259

DOI: 10.4000/appareil.2259

ISSN: 2101-0714

\section{Publisher}

MSH Paris Nord

\section{Electronic reference}

Martine Lefeuvre-Déotte, «Le travail de Rithy Panh : un appareil funéraire », Appareil [Online], Articles, Online since 29 March 2016, connection on 30 July 2020. URL : http://journals.openedition.org/ appareil/2259; DOI : https://doi.org/10.4000/appareil.2259

This text was automatically generated on 30 July 2020 .

\section{(c) (i) $\ominus$}

Appareil est mis à disposition selon les termes de la Licence Creative Commons Attribution - Pas d'Utilisation Commerciale - Pas de Modification 4.0 International. 


\title{
Le travail de Rithy Panh : un appareil funéraire
}

\author{
Martine Lefeuvre-Déotte
}

«Que demandent les morts? Qu'on pense à eux ?

Qu'on les libère en jugeant les coupables? ou veulent-ils qu'on comprenne ce qui a eu lieu?»

Rithy Panh ${ }^{1}$

1 Le réalisateur cambodgien, discrètement, l'affirme lors de ses entretiens : il est personnellement un survivant du génocide perpétré par les Khmers rouges entre 1975 et 1979 où plus de $20 \%$ de la population a été exterminée sous le régime de $\mathrm{Pol} \mathrm{Pot}^{2}$. Ce n'est que récemment, en 2011, avec la publication de son ouvrage coécrit avec Christophe Bataille, L'Élimination, que le public français a pu saisir ce qu'a été, précisément, la vie du jeune Panh entre 13 et 17 ans, puisqu'il a traversé ses années d'adolescence sous ce régime de terreur. J'affirmerai avec Tobie Nathan que «ce livre, [n]ous travaille, pénètre les méandres de [n]otre âme et s'assoit là, tout au fond comme un léopard à l'affût ${ }^{3}$ ». Si le lecteur reste à jamais frappé par les textes de Primo Levi ou d'Antelme, c'est à cette identique expérience qu'il se confronte avec l'écriture de Panh.

2 Nous avons été nombreux en France à prendre connaissance de la barbarie extrême des génocideurs cambodgiens par son travail cinématographique: Bophana, une tragédie cambodgienne (1996), S21, la machine de mort khmère rouge (2004), Duch, le maître des forges de l'enfer (2012), L'Image manquante (2013). Ce n'est que tardivement que Panh se livre à la première personne, raconte sa propre histoire à la fois par les images et l'écriture ${ }^{4}$. Il lui a fallu attendre, comme d'autres, le mi-temps de la vie pour briser sa loi du silence. Impossible de mettre en mots et en images le récit de sa famille; culpabilité d'être encore en vie face à cette extermination de masse. C'est donc bien à la frontière de la vie et de la mort que cherche à se tenir le survivant Panh. Pour extraire du néant les anonymes broyés par les Khmers rouges, les réinscrire dans l'humanité et pour se sauver lui-même: Panh traque, interroge et travaille les archives; inlassablement, continûment, il ne cesse de redonner la vie.

Ceux qui comme nous ont traversé ces épreuves sont morts une fois. Nous sommes des survivants. Nous revivons mais avec une part de mort en nous [...] L'art, la 
création, le cinéma redonnent du souffle à l'âme. Je suis mort, je renais. Mais je

renais avec la mort. En même temps, cette mort m'a construit ${ }^{5}$.

Les Khmers rouges utilisaient le novlangue, euphémisaient leurs exactions, ce n'est pas à une simple entreprise de mort qu'ils se livraient mais au kamtech, terme utilisé des milliers de fois par les membres du Parti, par Duch dans ses annotations des dossiers du centre d'extermination S21 dont il était responsable. En khmer, ce mot signifie non seulement tuer, mais « détruire puis effacer toute trace. Réduire en poussière [...] Qu'il ne reste rien de la vie, et rien de la mort. Que la mort elle-même soit effacée » (Panh \& Bataille, 2011, p. 105). Disparition de la disparition. Éliminer totalement les identités, falsifier les histoires, réduire en cendres les cadavres. C'est contre cette folie non seulement meurtrière mais « disparitionniste » que Rithy Panh s'élève, colossal défi ! La sociologie s'attaque toujours à la même question: qu'est-ce qui fait agir l'acteur? Comment expliquer que des individus accordent autant de temps, d'énergie, d'argent même pour mener à bien une action? ce que Bourdieu appelle illusio. Ce qui me retient ici, c'est de préciser quelques "ressorts» du travail de Panh. Ce survivant parle souvent de "sa boule d'énergie " qui le pousse inlassablement, malgré lui, dans sa volonté de savoir et d'inscrire dans la vie.

\section{Lutter contre la falsification de l'histoire}

4 Si nous avons connu en France les effets délétères du négationnisme et du révisionnisme face à la shoah, $\mathrm{Panh}^{6}$ doit s'attaquer à la fabrication d'un récit historique qui a longtemps nié la réalité du génocide organisé par le régime de Pol Pot. En 1991, les accords de paix signés à Paris entre les différentes factions politiques cambodgiennes, sous la menace des Khmers rouges de quitter la table des négociations et avec l'appui de la Chine, font disparaître le mot " génocide ». La machine à tuer n'a pas existé. Les Khmers rouges continueront à siéger à l'ONU jusqu'en 1993. Soko Phay Vakalis (2007, p. 3-4) remarque également qu'au Cambodge, ce n'est que depuis février 2009 que le génocide entre enfin dans les manuels d'histoire. Afin de gagner la paix, les institutions elles-mêmes tordent les mots pour aseptiser la réalité, antienne bien connue. Combien de temps a-t-il fallu en France pour que nos plus hauts représentants de l'État et l'histoire officielle reconnaissent qu'en Algérie entre 1954 et 1962, il ne s'agissait ni d'« événements », ni de «rétablissement de l'ordre » mais bien d'une guerre de libération des Algériens?

Décrire, nommer, comprendre précisément les actes et la volonté de l'Angkar (« organisation » en khmer), telle est déjà la volonté de Panh pour fissurer le mur du silence et du mensonge, lutter contre l'oubli, décortiquer avec minutie les rouages de la machine à tuer. Entre 1975 et 1979, une organisation parfaitement efficace et réfléchie, un "grand corps" pour reprendre la formule de Lefort, une famille dont les chefs numérotés, quasi invisibles, tiennent les rênes, conduit son entreprise révolutionnaire. Elle embrigade les jeunes paysans analphabètes, assène une pensée par slogans : «qui proteste est un ennemi, qui s'oppose est un cadavre »; « il faut détruire l'ennemi visible et aussi celui qui est invisible, l'ennemi dans sa pensée » (Panh, (2011), p. 121). L'Angkar interdit la religion, l'école, vide les villes et disperse les familles; elle s'attaque à tous ceux qui résistent à la révolution communiste: les citadins, les intellectuels, les bourgeois, les propriétaires et les membres trop modérés. L'idéologie du Parti cherche à restaurer la grandeur de la race khmère, celle d'Angkor et, dès 1970, "elle dicte le 
processus d'éradication planifiée des minorités nationales non khmères: les Chams musulmans, les Vietnamiens, les Chinois, les Laotiens et les Thaïlandais " (Kiernan, 1998). Le "nouveau peuple » doit être rééduqué à la campagne par "l'ancien peuple ", c'est-à-dire les paysans. L'Angkar planifie un crime de masse contre les ennemis de la révolution en les affamant, par des marches harassantes mais aussi en les éliminant. À Phnom Penh, Duch, le dirigeant du centre de torture et d'extermination S21 (196 bureaux de sécurité comparables existaient à cette époque), ne répond pas à la figure de la banalité du mal. Il obéit à des ordres précis et fait fonctionner avec un zèle absolu la machine à éliminer, à kamtech. Face aux mensonges, Panh ne cesse d'apporter des preuves.

Dans son documentaire Bophana, une tragédie cambodgienne, il s'attache précisément à l'histoire d'une "ennemie de la révolution» éliminée à S21. Bophana sort de l'anonymat, s'échappe des statistiques. Il lui redonne vie. Les génocideurs voulaient la réduire en poussière, il exhume sa photo, les centaines de pages écrites de sa main, il interroge ses proches; elle retrouve son identité. On saisit l'implacable organisation de la machine à tuer: photographier l'inculpé à titre de document, le déshumaniser, le réduire à l'état d'animal ${ }^{7}$, détruire sa mémoire, le contraignant sous la torture à avouer ses « fautes » face à l'Angkar, le transformer en coupable : la mort doit être juste. Si sa confession écrite signée par lui-même est jugée insatisfaisante, on le torture à nouveau jusqu'à ce que son récit corresponde à l'image de l'ennemi. À S21, les textes sont lus et annotés par Duch et ses supérieurs. De sept heures à minuit, des années durant, il souligne, annote, exige des précisions, des développements puis décide, un jour, de l'élimination. Dès 1970, lorsque l'Angkar s'attaque à un « ennemi ", elle n'informe de sa mort ni ses proches ni sa famille; elle ne restitue pas le cadavre. Elle peut même déterrer et brûler les ossements pour effacer toutes traces. L'élimination doit être tenue secrète. Bophana résiste à cette entreprise de destruction, d'altération de l'identité en continuant à écrire des lettres d'amour à son mari et en refusant de signer ses aveux de son nom officiel - elle signe sous celui de Séda, personnage du Râmâyana. Elle continue d'appartenir à l'humanité, elle reste animée par la passion, la culture, elle se livre encore au «crime» de l'amour et de la pensée comme Winston dans 1984. Restituer une biographie, c'est ne pas être complice de l'effacement.

\section{Le sens de la dette}

7 Une autre force anime Panh, je l'appellerai avec Marcel Mauss, l'obligation de rendre. Donner, restituer la vérité sur ce crime porté disparu certes, mais aussi, profondément, rendre à ses proches (son père, sa mère, le peintre Nath son ami) ainsi qu'aux inconnus éliminés, ce qu'ils lui ont donné, transmis. Panh doit obéir à une intraitable exigence à laquelle, d'abord pour une question de survie, il ne peut se dérober. Il doit obéir à la triple obligation du donner-recevoir-rendre. Ce lien, le seul qui l'unisse encore à ses parents défunts. C'est finalement dans son dernier film, L'Image manquante, que l'on saisit cette injonction.

Au milieu de la vie, l'enfance revient. C'est une eau douce et amère. Mon enfance, je la cherche, comme une image perdue. Ou plutôt, c'est elle qui me réclame [...] Avec de la terre et de l'eau, avec les morts, les rizières, avec des mains vivantes, on fait un homme [...] son costume est blanc, sa cravate sombre. Je voudrais le tenir contre moi, c'est mon père (Panh, 2013, p. 9-10) 

chapitres sa propre histoire et son entretien avec le génocideur Duch, est développée l' idée selon laquelle Duch ne correspond en rien à la figure d'Eichmann que dresse Arendt dans son rapport sur la banalité du mal (Arendt, 1966). Duch est un exécutant zélé qui met son point d'honneur à faire fonctionner la machine : «je ne discutais pas la voix du Parti. Je fonçais pour mon honneur. Si j'échouais, si je n'obtenais pas de confessions "camarade Duch, vous n'êtes plus à l'avant-garde ? Vous êtes usé" » (Panh, 2012). Tout est pensé à S21, jusqu'à la division des tâches: ceux qui comptent, appellent, soignent, interrogent, torturent, égorgent (les moins éduqués), enterrent. Les exécutants sont eux-mêmes contraints à l'exercice de la confession publique, donc à l'éventuelle élimination. L'Angkar voit tout, la machine orwellienne marche à plein régime, tous se surveillent, se dénoncent et participent au crime. Ils se tiennent, liés par le secret.

9 Face aux génocideurs, des individualités développent a contrario ce que Panh appelle la banalité du bien. Ce sont tous ces êtres qui résistent à l'entreprise d'élimination, qui se battent et combattent. Son père Panh Law, sa mère, le peintre Vann Nath, Bophana. Panh Law, homme cultivé, haut fonctionnaire de l'éducation nationale supporte difficilement l'entreprise de rééducation, c'est-à-dire le travail épuisant et la famine. Il décide un jour de ne plus s'alimenter, estimant que la nourriture distribuée avec une scandaleuse parcimonie par les Khmers rouges est indigne : «je ne mange pas ce qui ne ressemble pas à de la nourriture pour êtres humains» (2011, p. 99). Il périra rapidement dans la chambre même qu'il partage avec son fils puis sera enterré à la sauvette.

10 Panh Law qui jusqu'au bout refuse d'accepter le programme de l'Angkar, l'animalisation du vivant, au risque de sa vie, correspond exactement à ce que Panh appelle la « résistance du quotidien », la «banalité du bien ». Ce n'est que dans l'aprèscoup, en réfléchissant plus tard à ce comportement que Panh s'incline devant la force de son père qui ne plie pas, ne se soumet pas, n'obéit pas. La mère, par son dévouement extrême, son anticipation, sa connaissance des plantes, son affection pour les siens, sa débrouillardise force le respect. La banalité du bien, c'est cette mère qui garde quelques grammes d'or pour l'avenir. Alors que le jeune Rithy a échappé à la mort par dysenterie, il entend sa voix : «lève-toi, tu dois marcher ${ }^{8}$ ». Son père, comme sa mère lui transmettent la force de la résistance, la volonté de rester dans l'humanité. Le peintre Vann Nath, enfin, rescapé de S21, qui a gagné sa survie en peignant des portraits de Pol Pot admirés et commentés régulièrement par Duch, sa vie durant, cherchera à témoigner. Après la chute du régime, de mémoire, il représentera dans ses tableaux les scènes de tortures avec une infinie précision pour faire voir comment fonctionnait S21. Vann Nath veut aussi comprendre ; plus que juger, il est animé par la volonté de savoir. Dans S21, c'est lui qui conduit les discussions entre anciens geôliers et rescapés : où était la pensée ? qui réfléchissait ? pourquoi une telle barbarie ? La quête ultime de Nath et de Panh est de saisir les mécanismes de cette terreur; c'est là la portée universelle de leur travail : il ne s'agit pas seulement de témoigner du génocide cambodgien, plus fondamentalement, il convient de l'analyser pour éviter que de tels actes ne se reproduisent. C'est leur horizon. S'ils sont animés par cette volonté, ils ne croient guère à l'impact réel de leur quête, mais elle s'impose.

11 Panh a donc reçu beaucoup de la part de ceux qui sont morts: dignité, courage, fermeté, ténacité, il se doit de rendre à son tour, c'est-à-dire de résister sous toutes ses 
formes au travail d'élimination des Khmers rouges. C'est par les images et les mots qu'il comble le silence et redonne la vie. Il nous entraîne également dans ce cycle de l'échange. Qu'on relise l'injonction des dernières strophes de L'Image manquante ${ }^{9}:$ «Et cette image manquante, maintenant, je vous la donne, pour qu'elle ne cesse pas de nous chercher » (Panh \& Bataille, 2013, p. 69 $\left.{ }^{10}\right)$.

Le lecteur-spectateur-destinataire devient dépositaire d'une transmission, d'un savoir qu'il doit absolument rendre, faire circuler à son tour.

\section{Les pouvoirs spécifiques de l'appareil cinématographique}

13 Panh accorde à la caméra des facultés particulières et d'une grande importance. La littérature, la photographie, la peinture ne semblent pas assez complètes pour lui permettre de poursuivre sa recherche : traquer la vérité, analyser la folie meurtrière pour l'éliminer à jamais, donner vie. Seule la caméra, d'une certaine façon, est à la hauteur du défi. Existent chez Panh un Flaherty et un Rouch qui sommeillent. Pour pasticher le premier, Panh accorde à sa caméra toutes ses chances. Il n'hésite pas à filmer beaucoup ${ }^{11}$, ignorant le plus souvent au départ l'objet exact de son documentaire, comme s'il attendait la rencontre. Il se veut disponible, conduit par ceux et celles qu'il croise. Son terrain doit le surprendre, sinon à quoi bon? En 1989, dix ans seulement après son arrivée en France, Panh retourne en Asie pour tourner son premier film documentaire sur un camp de réfugiés cambodgiens en Thaïlande, Site 2: 180000 personnes vivant sur $4,5 \mathrm{~km}^{2}$. Il obtient une autorisation pour filmer trois jours, il n'a pas encore de sujet:

J'ai dit à tout le monde: Prenez une boite de dix minutes, baladez-vous dans le camp et filmez ce qui vous touche; mais surtout immergez-vous dans le camp, dans cette petite ville, une vraie société avec des chefs, la prison, l'école, l'hôpital, etc. Et pendant ce temps-là, je faisais du porte-à-porte, je cherchais un personnage. C'est ainsi que j'ai rencontré Yim Om. Elle faisait la sieste. (Panh, 2000)

Il négociera une nouvelle autorisation auprès des militaires thaïlandais, prétextant que toutes les prises étaient inexploitables, tant la lumière était forte. Il tient son sujet, le personnage de Yim Om, sa vie quotidienne de réfugiée, sa résistance banale pour rester dans l'humanité : ranger et nettoyer sa maison, organiser la vie de la famille. Rithy Panh multiplie les plans séquences, il rencontre ses personnages en les filmant et en les écoutant. Site 2 est le film de Yim Om au pays des camps de réfugiés, comme Nanook est un film de Nanook sur Nanook, comme l'affirmait Flaherty. L'œil de Panh ne voit rien, son oreille n'entend rien, c'est en filmant et en enregistrant des jours entiers Yim Om, en regardant et en visionnant les rushes, en discutant le soir avec elle qu'il saisit une sorte d'idéal-type à la Max Weber: le réfugié. Sa solitude, sa colère, sa peine, sa mélancolie, sa résistance dans ses gestes humbles pour conserver sa dignité. Yim Om parlant de son passé, des rizières, de sa vie de paysanne, du bonheur de la pêche dans les lacs, du village, de la liberté, de sa maison donnera à Panh le sujet d'un autre film, une fiction cette fois: Les gens de la rizière (1994). D'une certaine façon, il recrée l'univers passé de la paysanne. Yim Om tiendra d'ailleurs le rôle de la mère de cette grande famille structurée par la culture du riz et le combat difficile avec la terre. rencontre, le témoignage de la réfugiée Yim Om, mais relance également la quête, 
l'enquête sur l'histoire cambodgienne, ici, la paysannerie. Objet totalement méprisé et ignoré, si ce n'est par les films de propagande des Khmers rouges.

Pendant le tournage de Bophana, Panh retrouve les traces d'un gardien de S21. Il souhaite absolument éviter un face à face trop brutal entre ce dernier et le peintre Vann Nath, rescapé de ce centre de torture; ils ne doivent pas se rencontrer. Il faut protéger Nath et lui interdire, pour quelque temps, le lieu du tournage. Le peintre, sous un prétexte quelconque, revient à S21 et reconnaît l'ancien bourreau. Il le prend par les épaules et l'amène devant ses tableaux: "cette scène, je ne l'ai pas vue, on me l'a rapportée. Est-ce vrai ? Est-ce vraiment ainsi que se déroulaient les séances de torture? ${ }^{12}$ » Nath veut vérifier l'exactitude de ses représentations. Ne mentent-elles pas ? N'a-til pas travesti la réalité ? Sa production picturale répond-elle à l'exigence de vérité ?

17 Le peintre demande à Panh de filmer cette scène qui donne lieu à un plan-séquence et qui deviendra la matrice de S21. Cette rencontre inopinée entre le rescapé et le geôlier donnera la trame de S21. L'événement sur le tournage d'un film conduit, là encore, à l'élaboration d'une autre production. Pour mener son enquête quasi policière, Panh joue avec les pouvoirs spécifiques de l'appareil ; pour $\$ 21$, pendant 3 ans, régulièrement, il revient à Phnom Penh filmer les gardiens et les quelques survivants. Deux techniques pour retrouver avec exactitude la mémoire des lieux et des exactions : confronter les génocideurs aux archives, les filmer face aux multiples photos et aux cahiers d'aveux, sur le temps long, introduire le trouble et s'attaquer à la mémoire du corps. Comme si le corps, lui, n'oubliait pas, comme si la caméra allait bien finir par dérober les images "vraies ». Faire appel à la routine du quotidien qui finira par étouffer le mensonge. À des mois d'intervalle, les geôliers rejouent les actions qui ponctuaient leurs journées : aller chercher un inculpé, crier les ordres, lui mettre un bandeau, le ligoter, lui faire traverser le couloir en le tenant par les épaules, l'amener à l'interrogatoire. La caméra traque les corps qui retrouvent la mémoire de cette histoire faite corps. Panh l'affirme souvent, il n'est pas au sens propre un historien, ses outils de recherche sont différents: pour lui, seule la caméra permet cette confrontation entre l'enquête et l'archive. Un exemple, dans Duch, le maitre des forges de l'enfer, le responsable de S21 affirme qu'il n'a jamais interrogé un détenu, sa seule mission était d'enseigner la théorie. Panh le filme alors regardant les images d'un gardien de S21 qui décrit à l'écran comment Duch intervenait dans les interrogatoires. Panh attend, à la caméra, la réaction de l'imposteur.

18 L'appareil cinématographique devient une sorte de "détecteur de mensonges », chacun devient le jouet de la technique et doit s'expliquer, se prononcer face aux images, aux autres aveux filmés. Cet appareil, évidemment, peut échapper au réalisateur qui n'en maitrise pas tous les effets. Duch, à son tour, utilise les pouvoirs de l'appareil. On sait qu'il a abandonné le bouddhisme et s'est converti au christianisme, il est devenu évangélique; dans sa cellule, les dernières images du documentaire (Panh, 2012) le montrent priant et communiant. Il souligne que « pour Bouddha, le karma dépend des actes. Dans le catholicisme, Dieu te pardonne; nous offrons à Dieu non un poulet, mais notre cœur meurtri, notre profond remords. Je vais vers le christianisme, affirme Duch, parce que j'ai trouvé le chemin du véritable amour ».

19 Le maître des aveux de S21 utilise la caméra de Panh comme moyen de livrer à la face du monde une longue confession destinée assez essentiellement au Dieu des chrétiens, il espère bien obtenir son pardon, le sauvetage de son âme. Ne serait-ce pas ce qui explique in fine cette étrange confiance accordée au cinéaste? Au début de sa rencontre 
avec Duch (il le filmera des centaines d'heures), alors que le réalisateur lui explique son projet : lui laisser la possibilité d'expliquer en détail le processus de mort dont il est l'organisateur, le génocideur confie, exalté «j'ai eu une révélation, ce matin, pendant ma prière. J'ai été submergé. J'ai compris : il faut que je vous parle. » J'ai répondu : «je ne demande que ça. » " (Panh \& Bataille, 2011, p. 24).

Étrange utilisation de cet appareil qui pour l'un fait advenir l'aveu, traque le mensonge et qui, pour l'autre, œuvre à gagner la rédemption. Si Panh ne peut approcher Duch que caméra à la main, canalisant ainsi sa propre violence - « le cinéma [...], je crois qu'il me tient les poings dans les poches " (Panh \& Bataille, 2011, p. 16) -, Duch l'utilise à de tout autres fins : obtenir le pardon de Dieu, gagner son Salut. Mais encore, l'appareil, c'est-à-dire le cinéma ne tient-il pas souvent lieu de linceul chez Panh? On le sait, ce qui reste insupportable avec la disparition, cette dissolution de la vie et de la mort, pour les proches comme pour la société tout entière, c'est la quasi-impossibilité de faire le deuil de ces «ni morts, ni vivants ». Déjà parce qu'aucun rituel funéraire n'a été organisé pour « la paix des vivants » (L. V. Thomas). Lorsque le réalisateur cambodgien relate dans L'Élimination (2011) les funérailles de son père, il insiste sur l'extrême célérité de la mise en terre, qui ne répond en rien aux exigences de la tradition. Le corps a été rapidement roulé dans un drap blanc conservé par la mère, puis dans une feuille de tôle et recouvert de terre avant la nuit à cause de la chaleur (Panh \& Bataille, 2011, p. 101). La mère refusera d'accompagner son mari, seule la grande sœur assistera à ce simulacre d'enterrement. Mais la mère, cette nuit-là, contera à son fils Rithy les funérailles du père, telles qu'elles auraient dû être.

Tu vois, au premier rang, devant le beau cercueil de bois, il y a les représentants des élèves; les instituteurs; puis les représentants du ministère, qui marchent en portant des couronnes de fleurs; la famille suit. Nous sommes tous là rassemblés

[...] Nous voici à ses côtés. Silencieux. Aimants. (Panh \& Bataille, 2011, p. 101-102)

Cet « enterrement de mots", selon la formule de Panh, dure la nuit entière et, sur « ce frêle esquif, le départ de mon père m'a semblé moins pénible. Mais à l'aube, nous étions seuls » (Panh \& Bataille, 2011; p. 102).

Les mots et les images deviennent alors totalement nécessaires pour enterrer dignement les morts. Là encore, Panh renoue le fil avec les défunts, paie sa dette en cherchant à les ensevelir honorablement par le biais de sa caméra. Bophana, une tragédie cambodgienne ou L'Image manquante en sont des exemples probants. À la fois, il redonne la vie, une histoire, une mémoire, une dignité aux disparus mais aussi il les ensevelit.

Le travail littéraire et cinématographique de Panh, s'il cherche à rétablir la vérité, analyser le génocide cambodgien, lutter contre l'effacement, donner la vie, agit aussi comme un rituel de deuil, pour lui-même et pour la société cambodgienne en son entier, cherchant à éviter que les survivants ne sombrent dans la folie hantés par leurs spectres. «Je suis venu vers le cinéma pour me sauver, ce n'est même pas le moyen pour m'exprimer ; c'est mourir ou faire ce film ${ }^{13} »$. 


\section{BIBLIOGRAPHY}

Hannah Arendt (1996), Eichmann à Jerusalem. Rapport sur la banalité du mal [1963], Paris, Gallimard, [1963].

Rithy Panh (1996), Bophana, une tragédie cambodgienne, film documentaire.

- (2000), La parole filmée. Pour vaincre la terreur, film documentaire, Paris, Éditions Montparnasse.

- (2004), S21, la machine de mort khmère rouge, film documentaire, Paris, Éditions Montparnasse

- (2011), avec Christophe Bataille, L'Élimination, Paris, Grasset.

- (2012), Duch, le maître des forges de l'enfer, film documentaire, Paris, Editions Montparnasse

- (2013) avec Christophe Bataille, L'Image manquante, Paris, Grasset.

Soko Phay-Vakalis, (2007) Rithy Panh, l'éveilleur de conscience. À propos du film Le papier ne peut pas envelopper la braise, http://www.espacestemps.net/auteurs/soko-phay-vakalis/

Bertrand Tavernier (2002), Entretien avec Rithy Panh à propos de S21, Paris, Éditions Montparnasse, 2002.

\section{NOTES}

1. Panh \& Bataille, 2011, p. 19

2. 1700000 personnes sur une population estimée à 7900000 habitants. Cf. Ben Kiernan, Le génocide au Cambodge 1975-1979. Race, idéologie et pouvoir, Paris, Gallimard, 1998.

3. Voir le blog de Tobie Nathan, https://tobienathan.wordpress.com/

4. Essentiellement L'Élimination et le film L'Image manquante.

5. Journal La Croix, entretien avec Rithy Panh par Jean-Claude Raspiengeas, à propos de L'Image manquante, 8/10/2013.

6. Cf. également le travail historique de Ben Kiernan, cité plus haut.

7. «Le prisonnier : je le regardais comme une bête ", affirme un geôlier dans S21. " On considère leurs vies comme des déchets ", avait avoué Duch.

8. France culture, 2012, émission À voix nue, entretien avec Christophe Bataille et Rithy Panh 16/05/

9. L'Image manquante est à la fois un film et un texte d'une forte charge poétique.

10. Je souligne.

11. "Je tourne beaucoup et la caméra numérique offre une liberté incroyable; il y a beaucoup de rushes et j'ai besoin de temps pour les regarder [...] Pour la terre des âmes errantes, il y avait 140 heures de rushes. On est passé à 10 heures, puis à 3 . On a fait une version de $1 \mathrm{~h} 20$ qui était catastrophique. Une des meilleures versions est celle de 1 h 55 ». Cf. Rithy Panh, La parole filmée. Pour vaincre la terreur, (2000), Paris, Montparnasse, 2000, p. 27.

12. On peut découvrir ces scènes dans Bophana, une tragédie cambodgienne.

13. Rithy Panh dans Tavernier (2002). 


\section{ABSTRACTS}

The Cambodian director and writer, Rithy Panh, born in 1962 in Phnom Penh, is a survivor of the genocide perpetraded by the Pol Pot regime (1975-1979). The Khmer Rouge organized a mass crime ( $20 \%$ of the population were exterminated). The target, they said, were the enemies of the revolution: the non-Khmers, the intellectuals, the owners, the city people, the middle-class. Their aim was not only to eliminate them but to make them disappear - kamtech in Khmer means not only to kill but to erase all traces, to reduce to ashes, so as to leave nothing of life or death. This crime itself long disappeared; in 1993, the Khmers Rouges were still acknowledged by the UN. How can a society survive to this monstrous oblivion, to this denial, without sinking into madness? The literary and film work of Panh not only endeavours to restore, 20 years along, the truth, methodically, implacably, to flight lies, to bring life to those who have no trace, no story, so as to allow them to a decent grave. By setting them in a narrative and in pictures, Panh allows, in his own way, a mourning ritual for all those who, alike the Mothers of the May Square in Argentina, had tremendously been deprived of it.

Le réalisateur et écrivain cambodgien Rithy Panh, né en 1962 à Phnom Penh, est un survivant du génocide perpétré par le régime de Pol Pot (1975-1979). Les Khmers rouges ont organisé un crime de masse (20\% de la population a été exterminée). La cible, affirmaient-ils, était les ennemis de la révolution : les non-Khmers, les intellectuels, les propriétaires, les citadins, les bourgeois. Leur but n'était pas seulement de les éliminer mais de les faire disparaître, kamtech en khmer signifie non seulement tuer, mais effacer toutes traces, réduire en poussière afin qu'il ne reste rien ni de la vie, ni de la mort. Ce crime a lui-même longtemps disparu : en 1993 encore, les Khmers rouges étaient reconnus par l'ONU. Comment une société peut-elle survivre à cet oubli monstrueux, à ce déni sans sombrer dans la folie ? Depuis plus de 20 ans, l'œuvre littéraire et cinématographique de Panh non seulement s'attache à rétablir la vérité, méthodiquement, implacablement, à lutter contre le mensonge, mais aussi à redonner vie aux sans traces et aux sans récit, permettant ainsi de les ensevelir dignement. En les fixant dans une narration et des images, Panh permet, à sa mesure, un rituel de deuil. Il organise des funérailles à tous ceux qui, telles les Mères de la Place de Mai en Argentine, en avaient été effroyablement privés.

\section{INDEX}

Mots-clés: Panh, Khmers rouges, génocide, disparition, déni, rituel funéraire, caméra

\section{AUTHOR}

\section{MARTINE LEFEUVRE-DÉOTTE}

Maître de conférences honoraire à l'université de Caen, Département de sociologie 